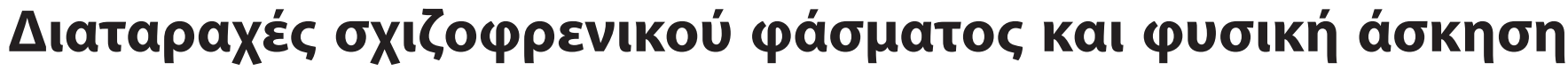

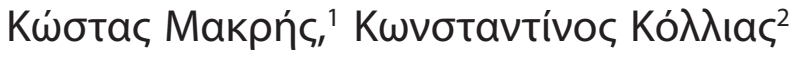

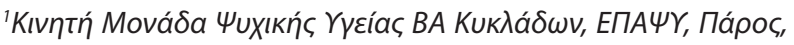

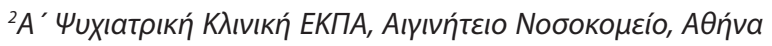

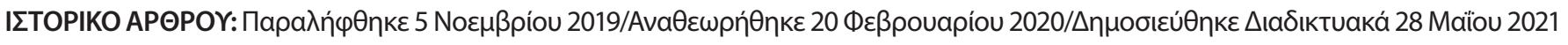

\section{ПЕРІАНЧH}

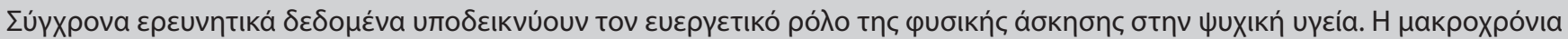

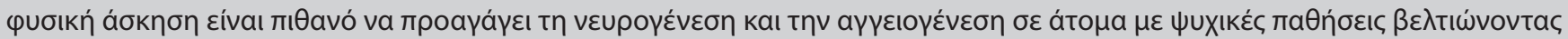

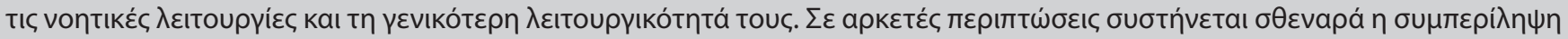

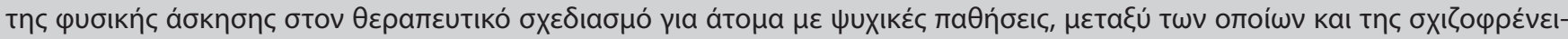

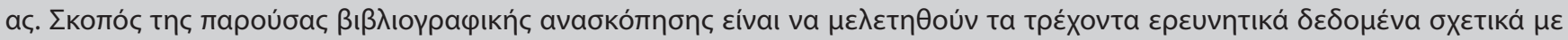

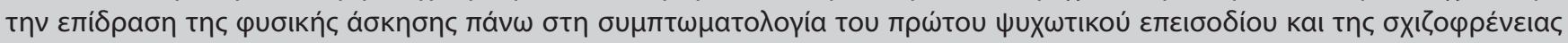

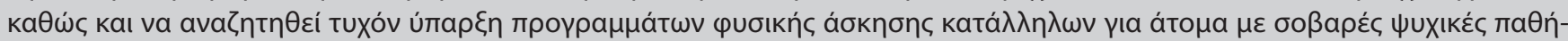

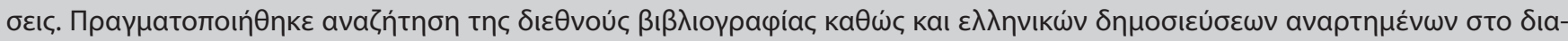

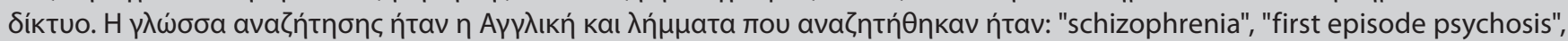

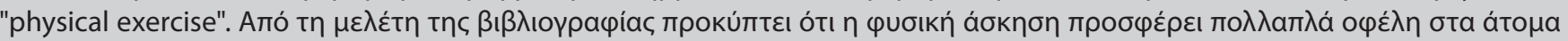

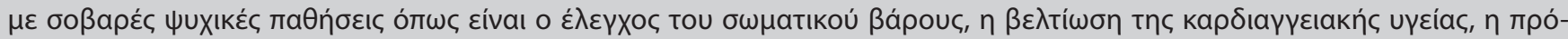

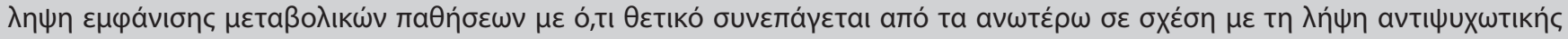

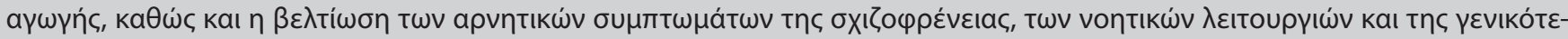

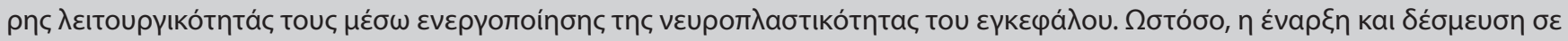

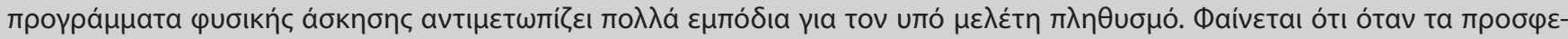

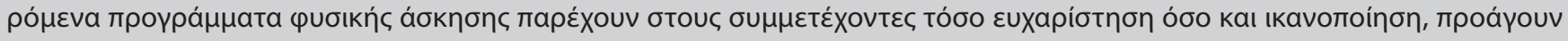

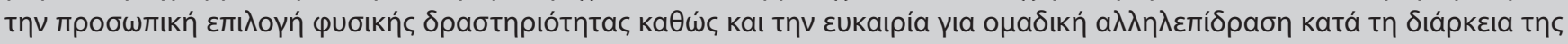

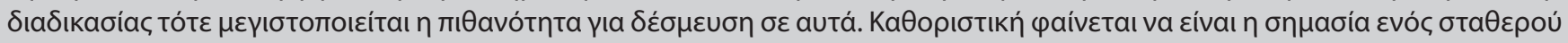

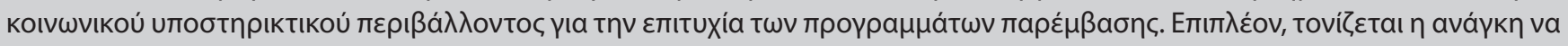

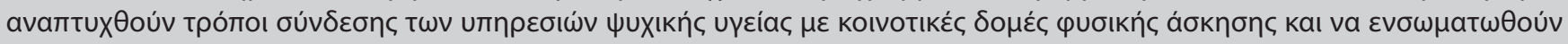

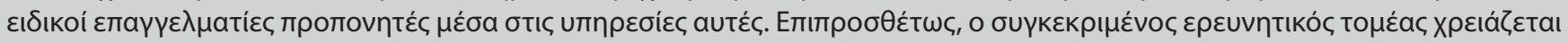

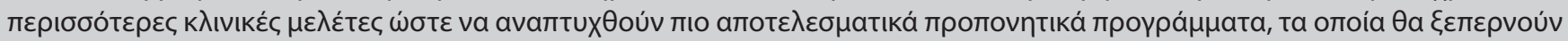

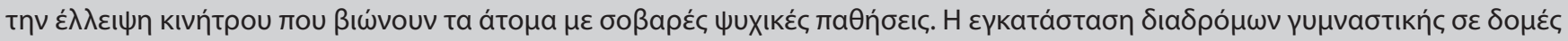

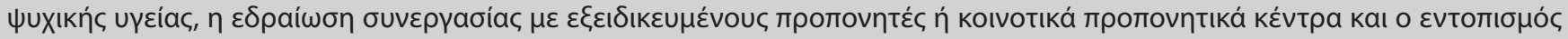

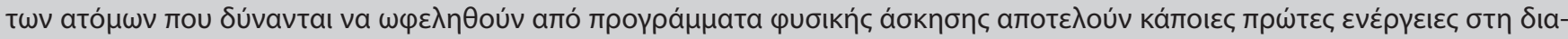

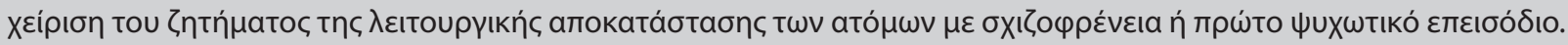

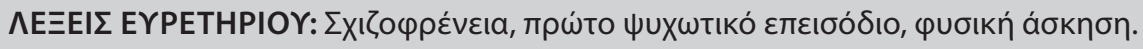




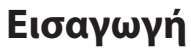

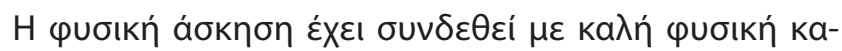

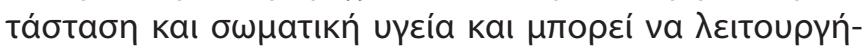

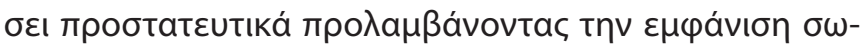

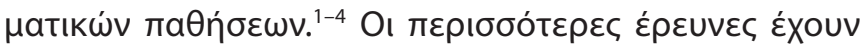

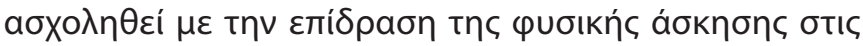

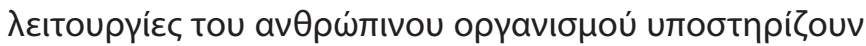

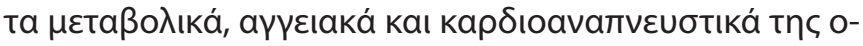
$\varphi \dot{\varepsilon} \lambda \eta{ }^{1,5-9}$

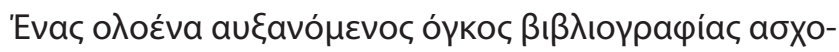

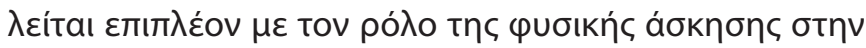

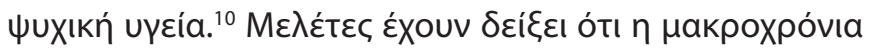

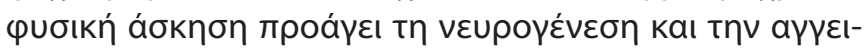

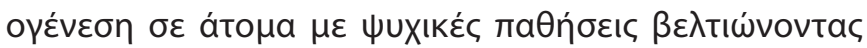

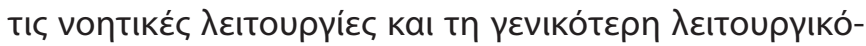

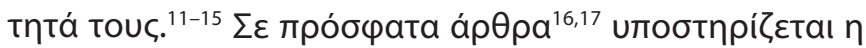

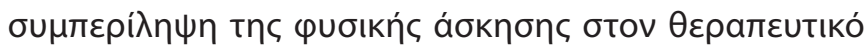

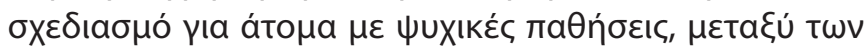

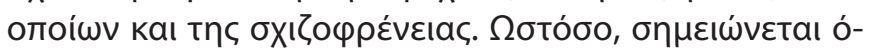

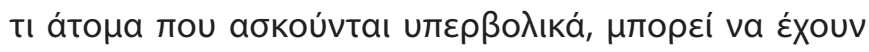

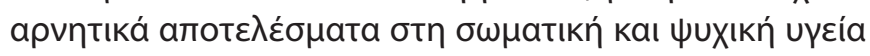
touc. ${ }^{18-20}$

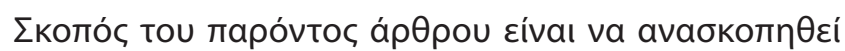

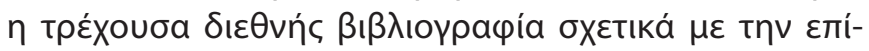

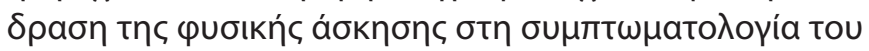

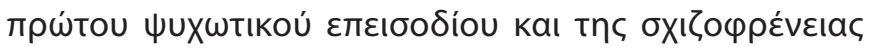

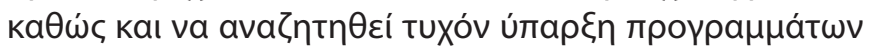

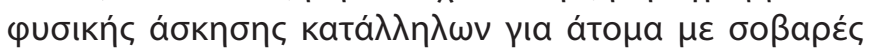

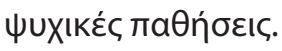

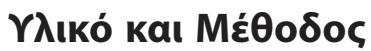

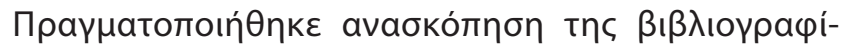

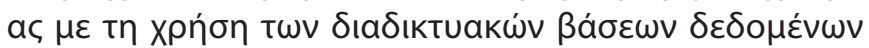

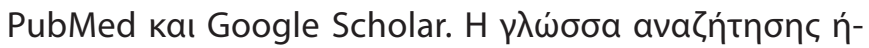

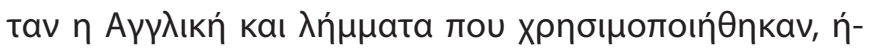
tav: "schizophrenia", "first episode psychosis", "physical

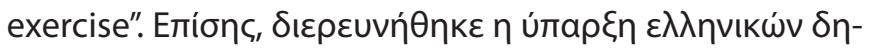

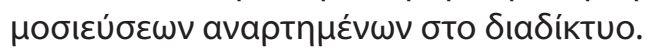

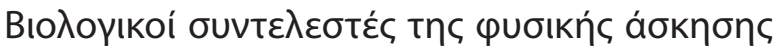

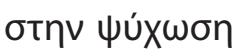

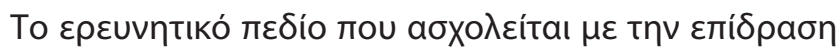

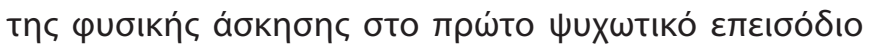

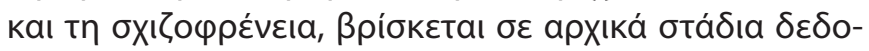

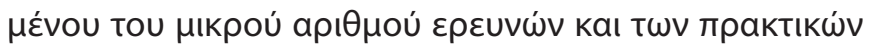

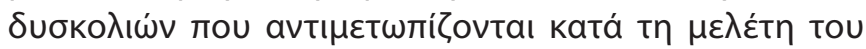

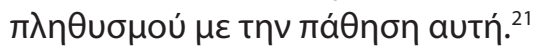

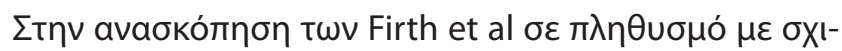

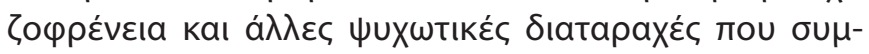

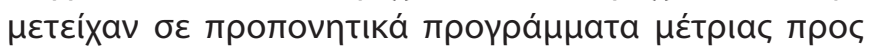

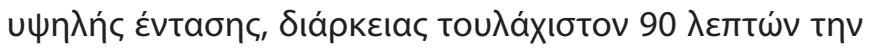

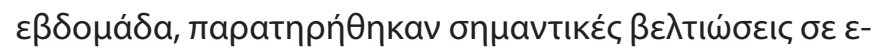

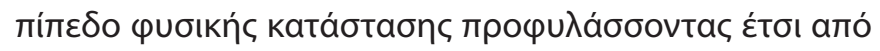

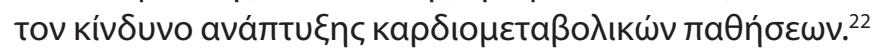

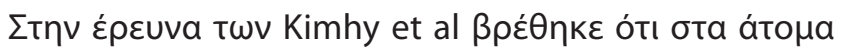

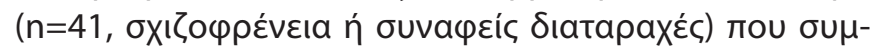

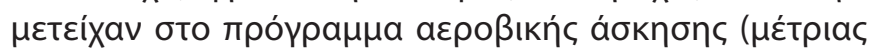

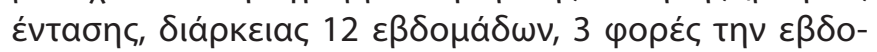

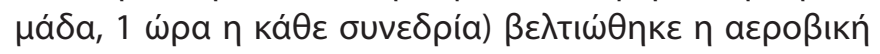

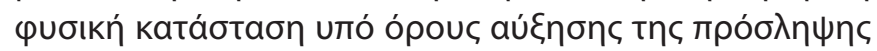

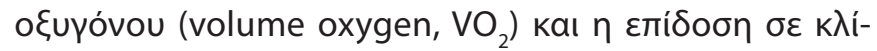

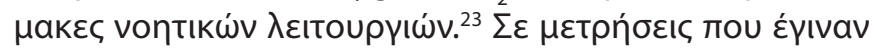

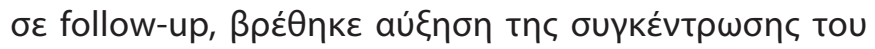

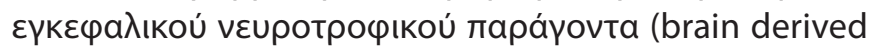

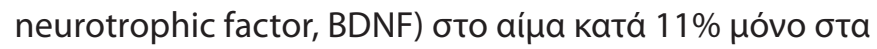

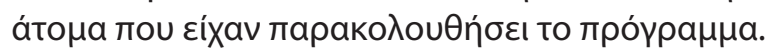

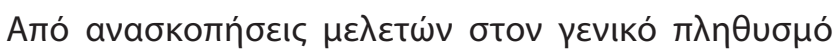

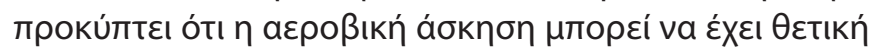

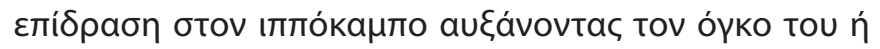

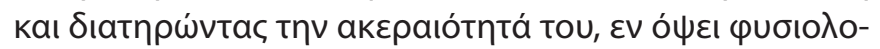

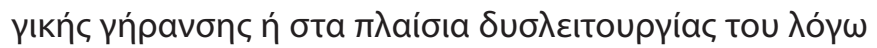

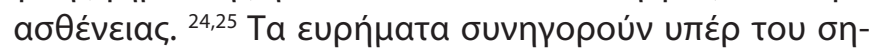

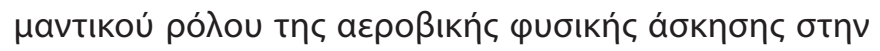

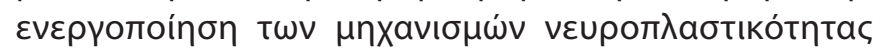

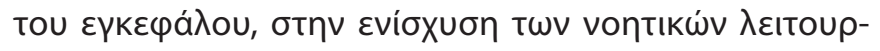

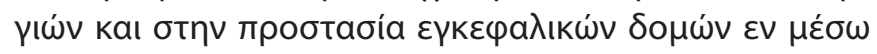

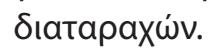

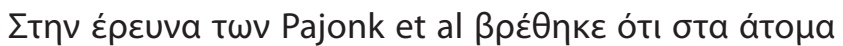

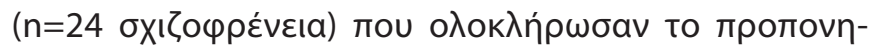

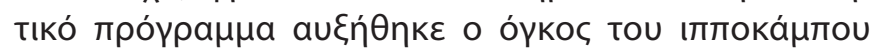

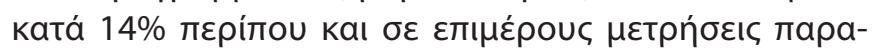

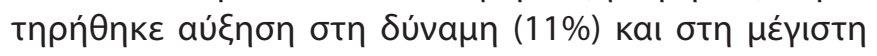

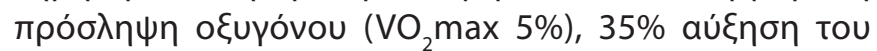

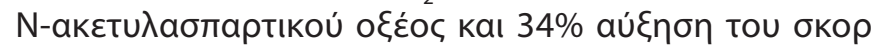

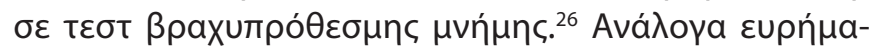

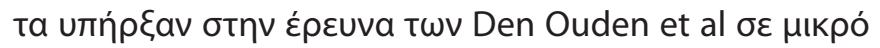

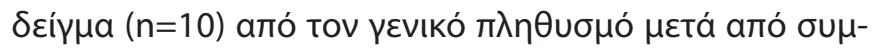

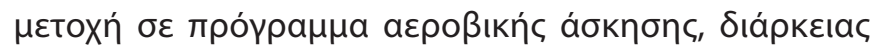

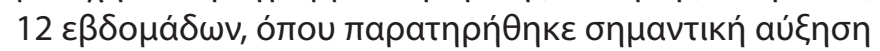

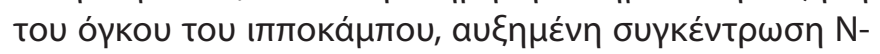

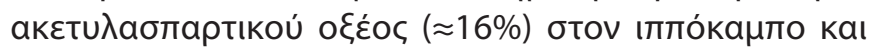

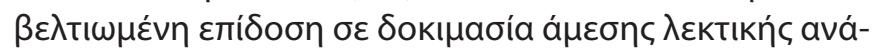

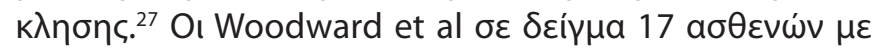

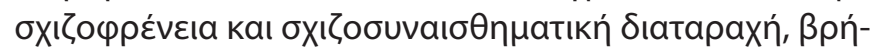

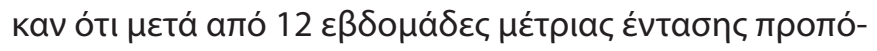

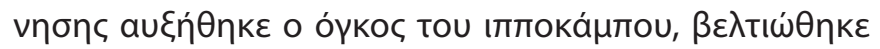

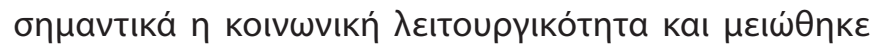

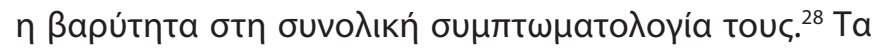

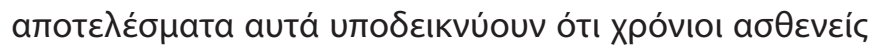

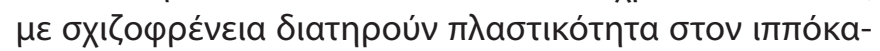

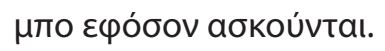

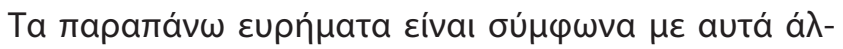

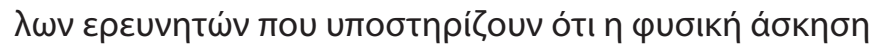




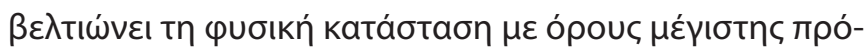

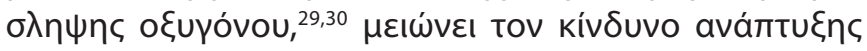

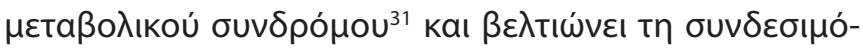

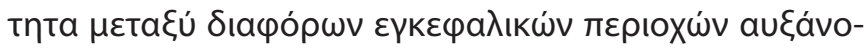

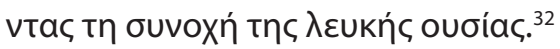

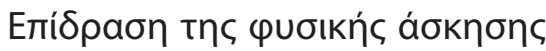

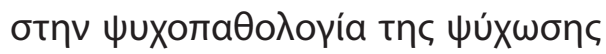

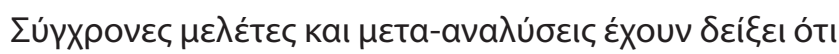

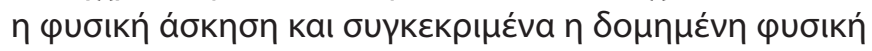

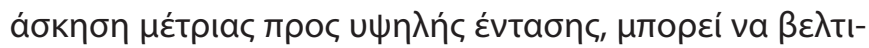

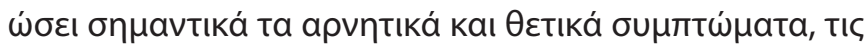

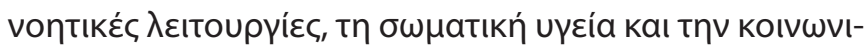

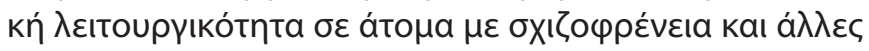

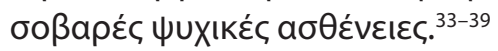

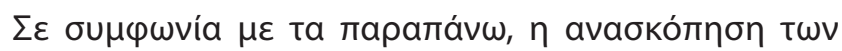

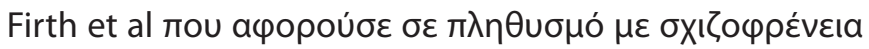

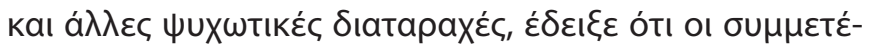

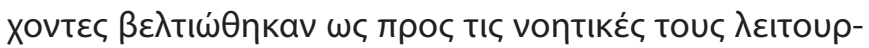

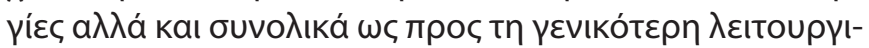
Kótntá touc. ${ }^{22}$

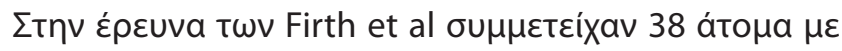

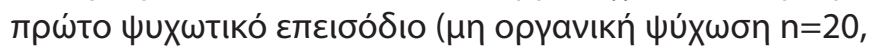

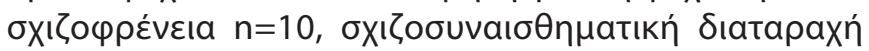

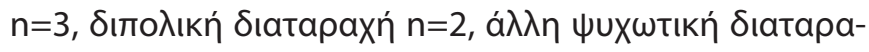

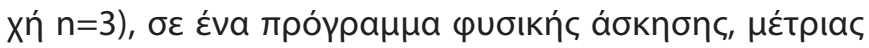

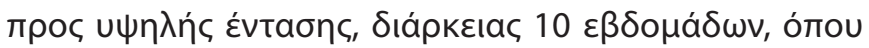

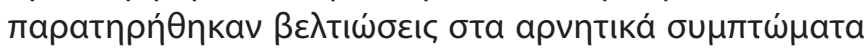

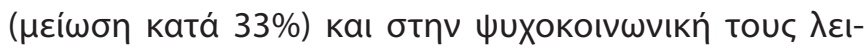

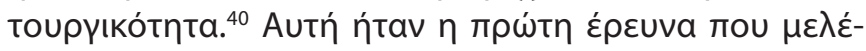

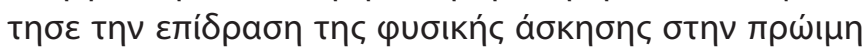

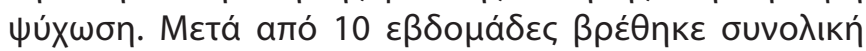

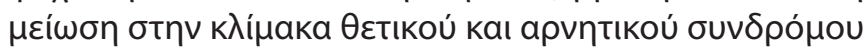
(Positive and Negative Syndrome Scale, PANSS) katá $27 \%$.

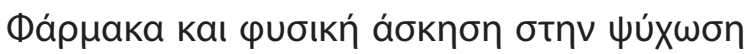

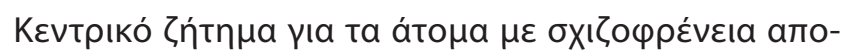

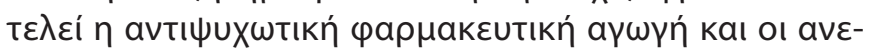

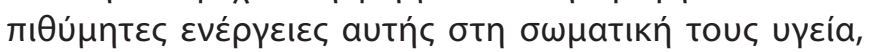

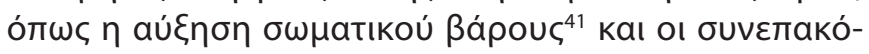

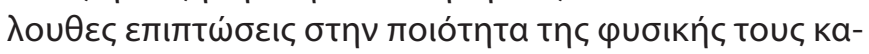
táotaonc.

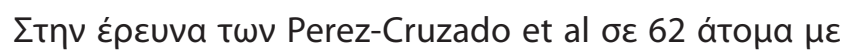

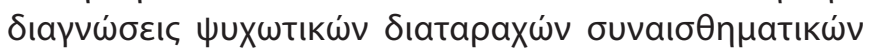
kal un (F20-F22/F24/F25/F28-F31/F32.3/F33.3 katá

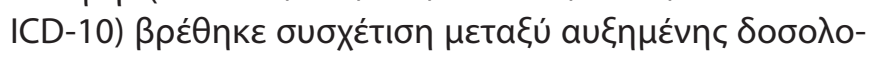

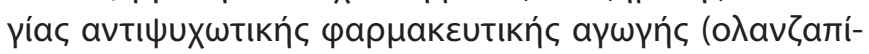

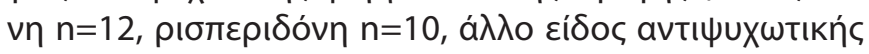

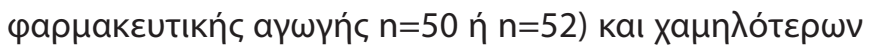

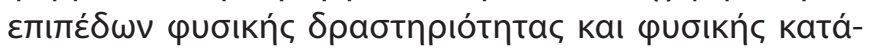
otaonc. ${ }^{42}$

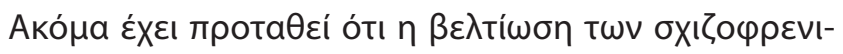

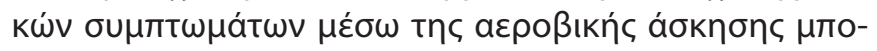

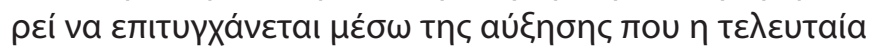

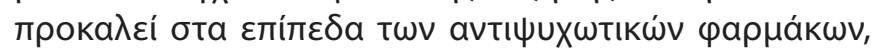

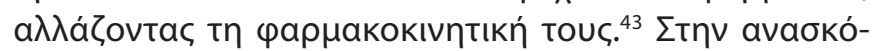

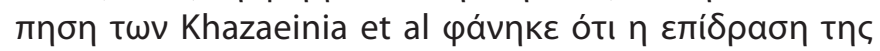

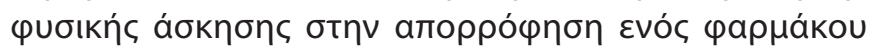

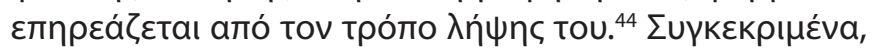

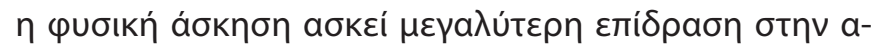

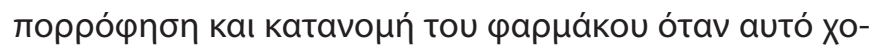

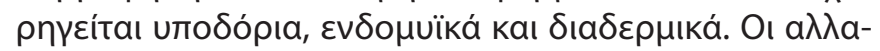

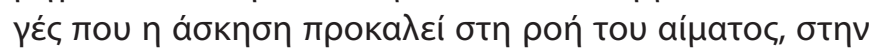

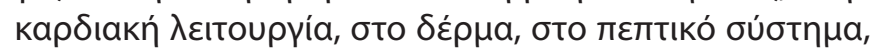

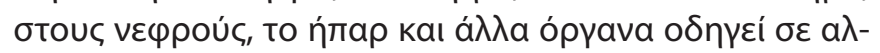

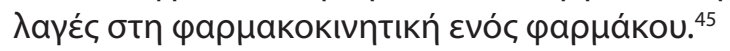

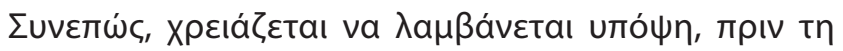

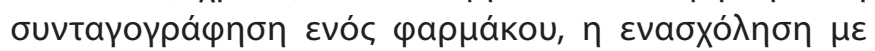

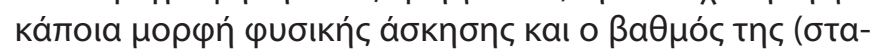

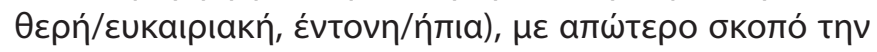

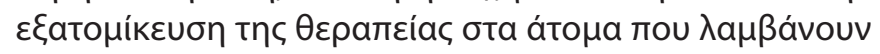

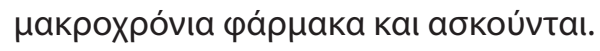

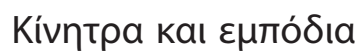

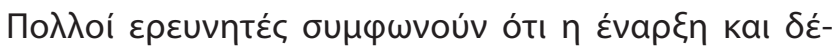

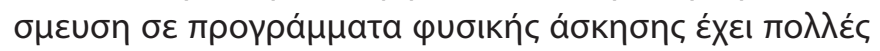

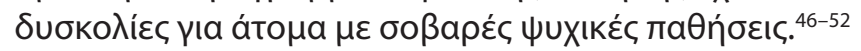

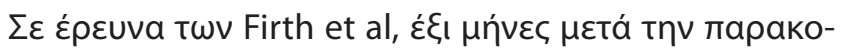

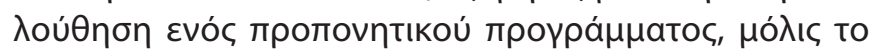

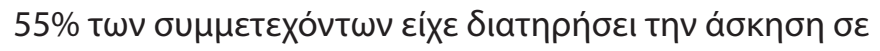

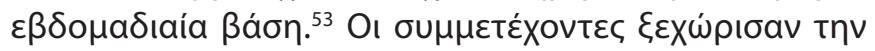

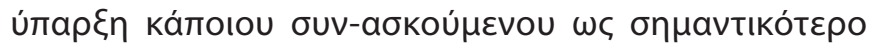

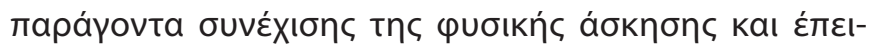

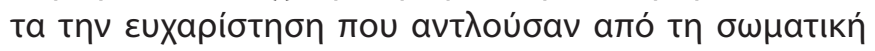

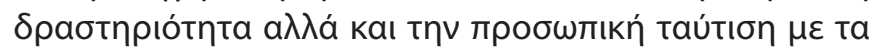

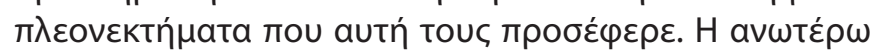

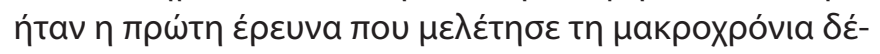

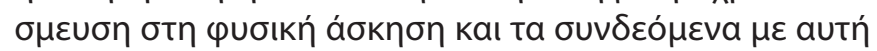

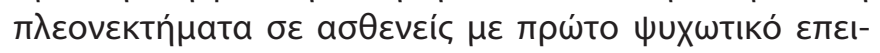

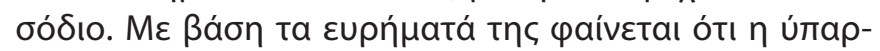

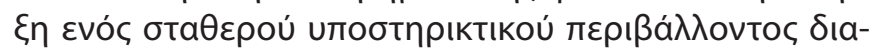

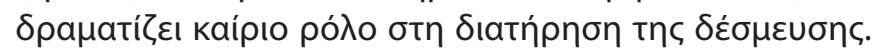

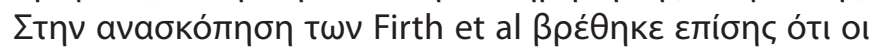

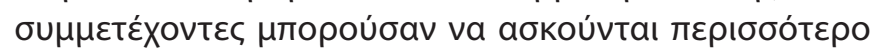

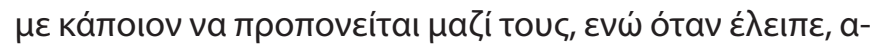

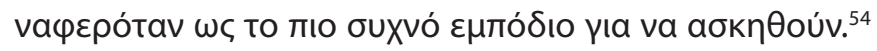

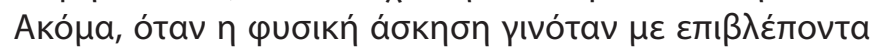

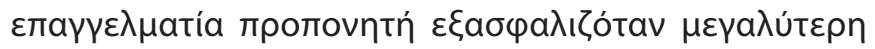

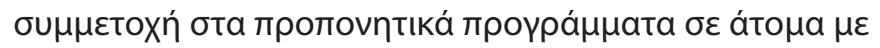

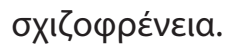

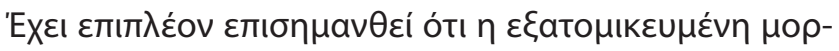

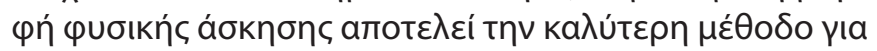

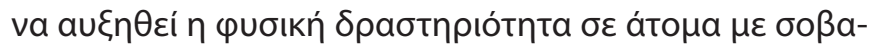




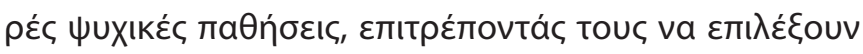

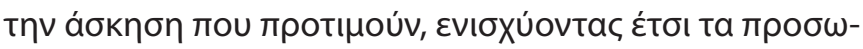

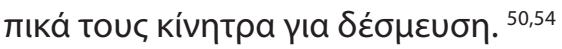

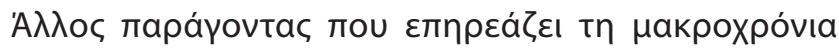

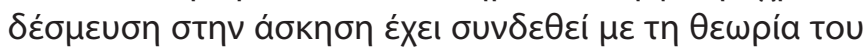

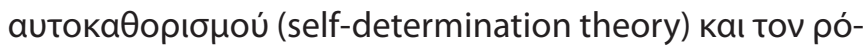

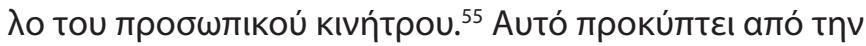

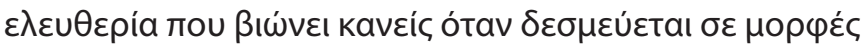

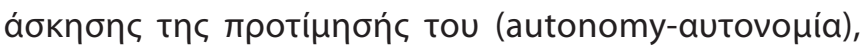

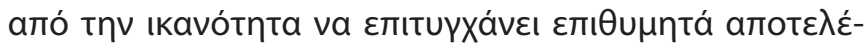

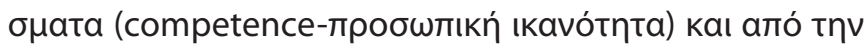

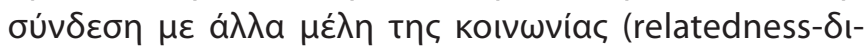

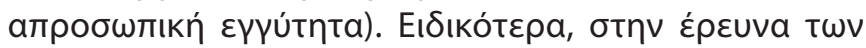

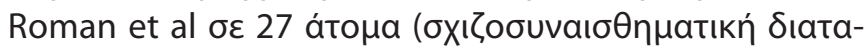

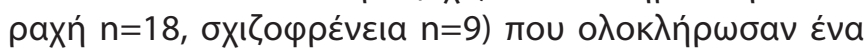

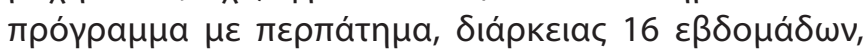

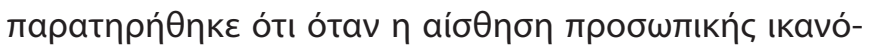

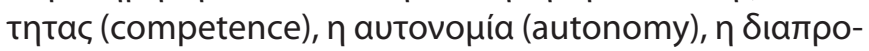

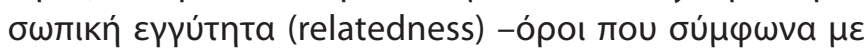

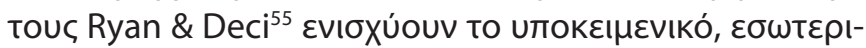

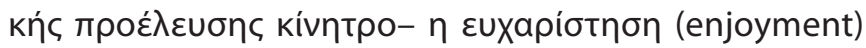

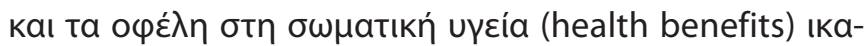

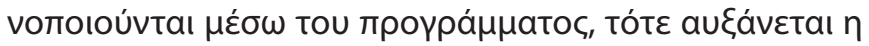

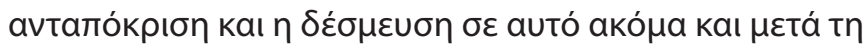
$\lambda$ ń£ฤ tou. ${ }^{56}$

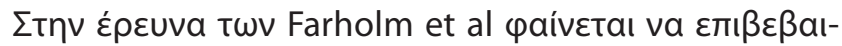

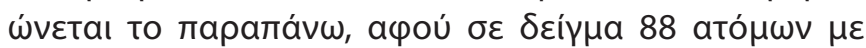

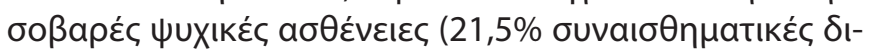

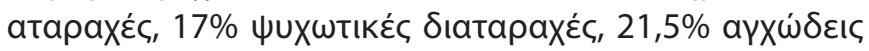

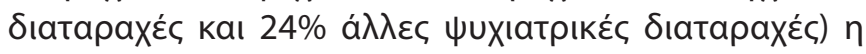
$\pi \lambda \varepsilon ı$

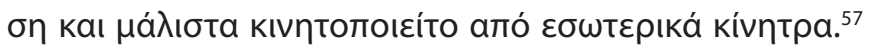

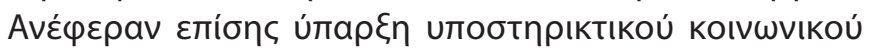

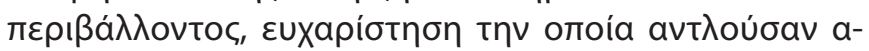

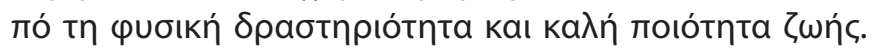

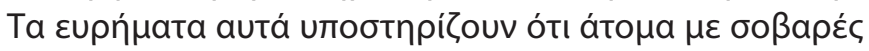

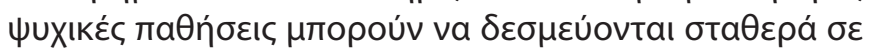

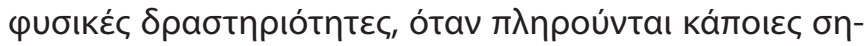

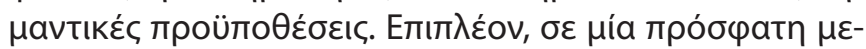

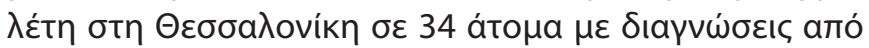

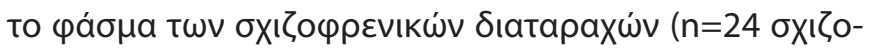

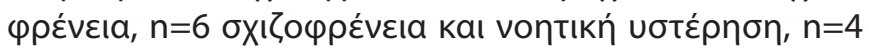

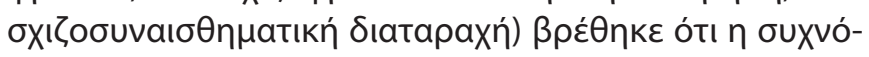

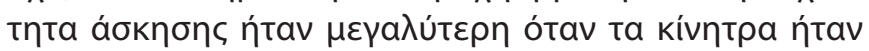

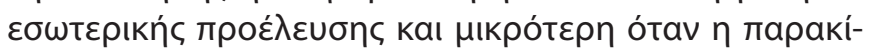

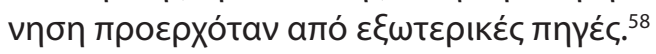

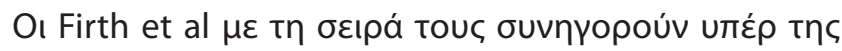

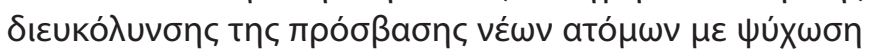

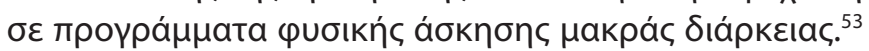

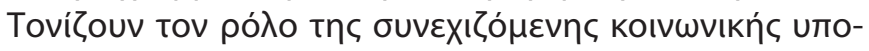

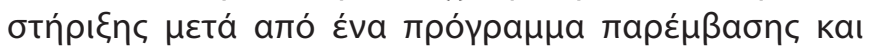

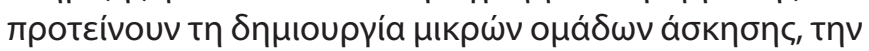

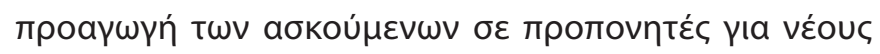

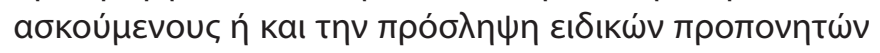

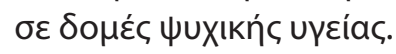

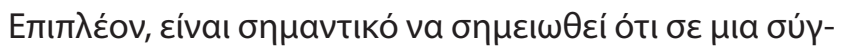

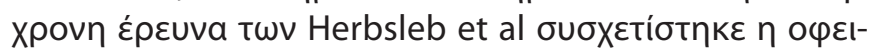

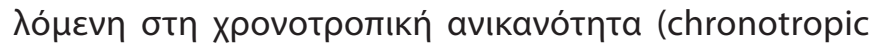

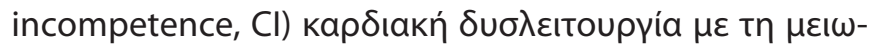

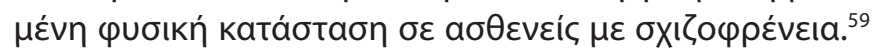

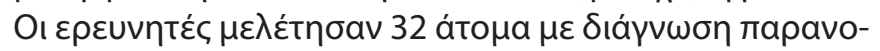

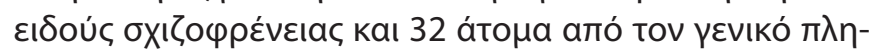

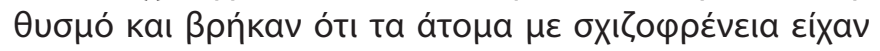

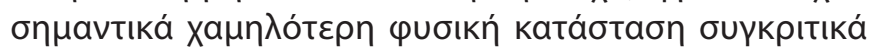

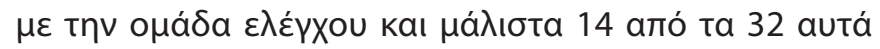

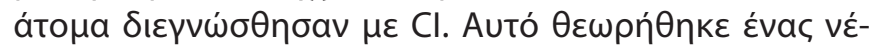

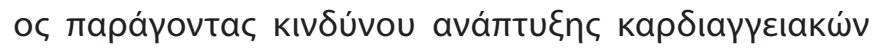

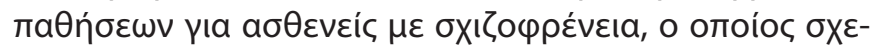

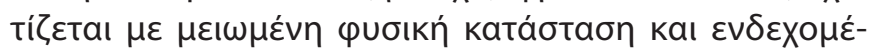

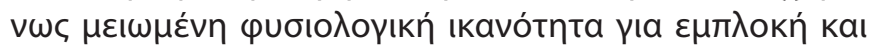

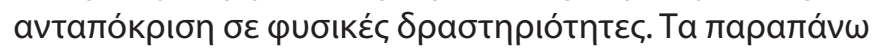

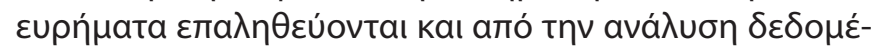

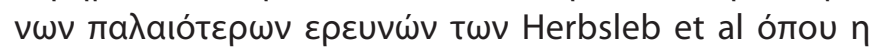

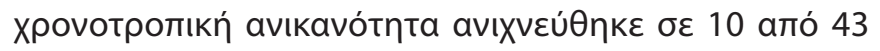

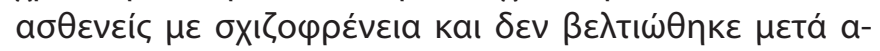

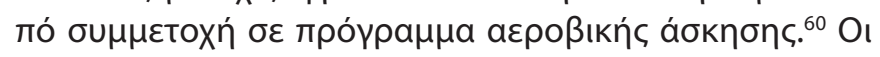

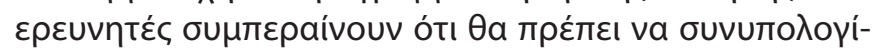

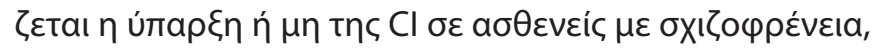

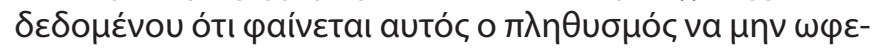

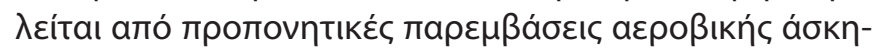

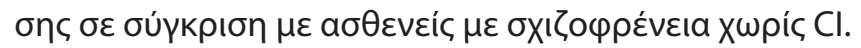

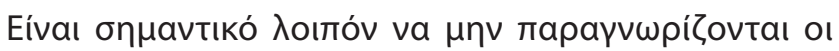

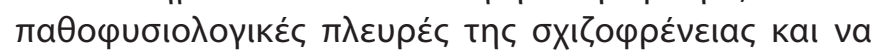

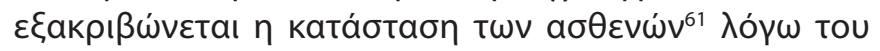

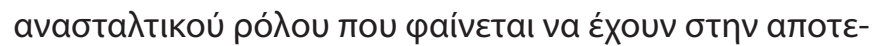

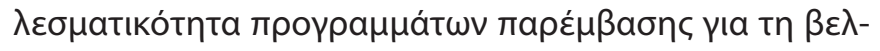

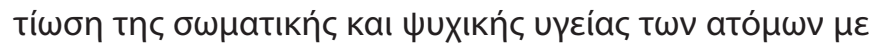

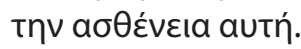

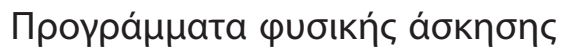

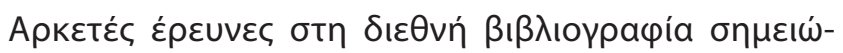

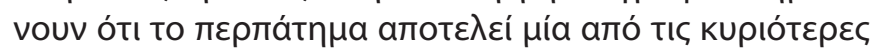

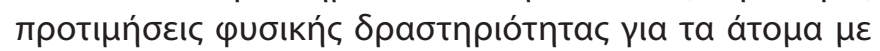

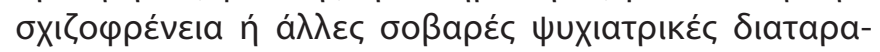
$X \chi^{\prime}{ }^{46,62,52}$

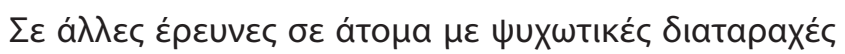

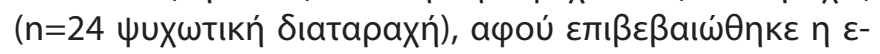

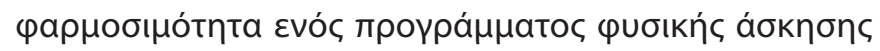

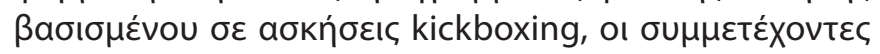

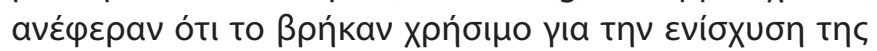

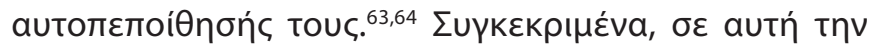

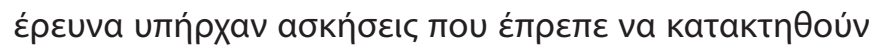

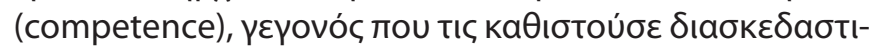

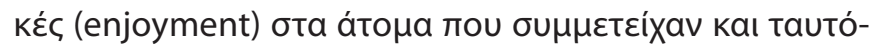




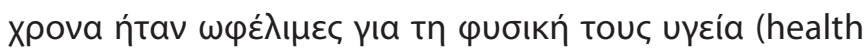

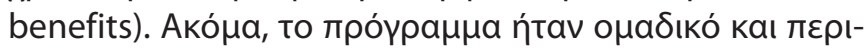

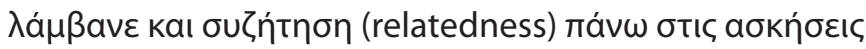

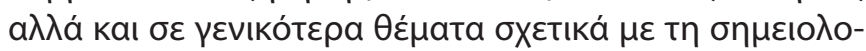

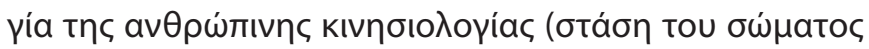

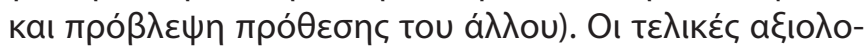

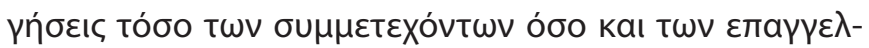

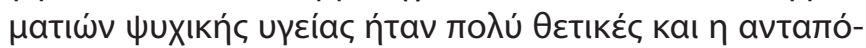

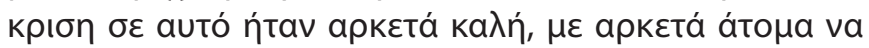

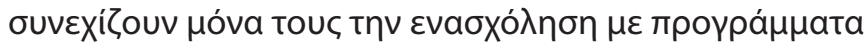

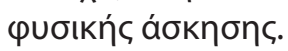

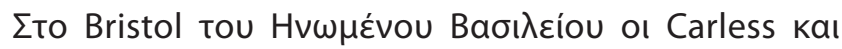

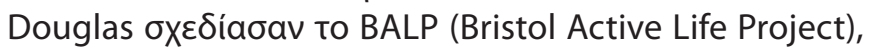

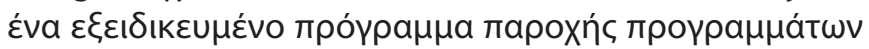

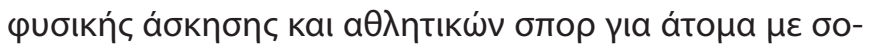

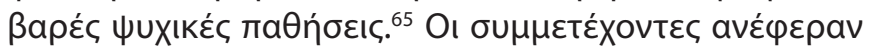

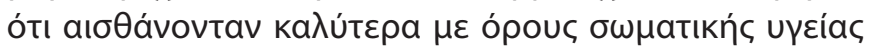

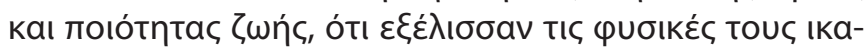

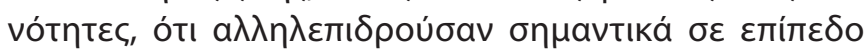

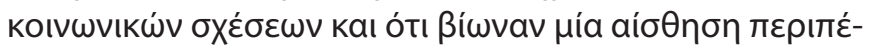

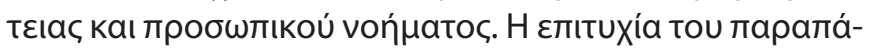

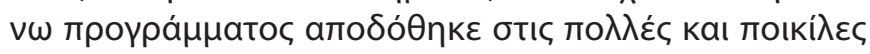

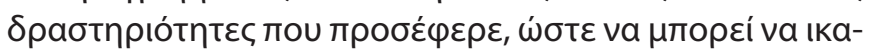

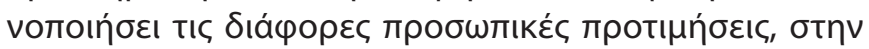

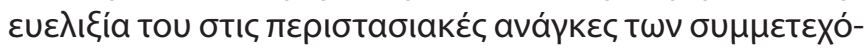

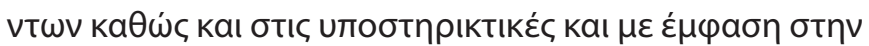

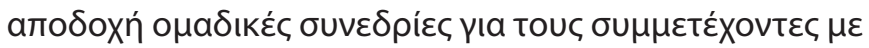

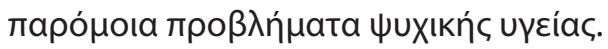

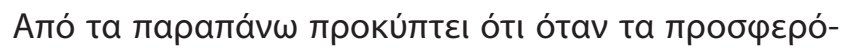

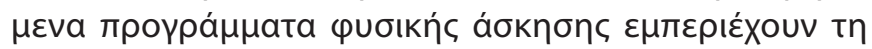

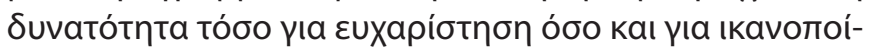

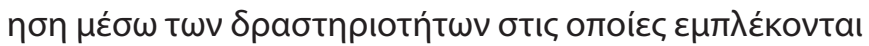

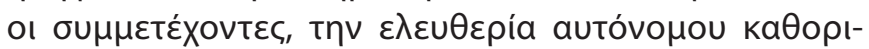

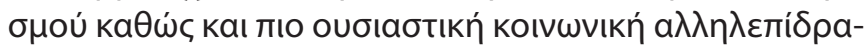

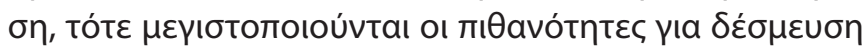

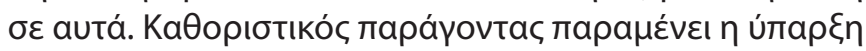

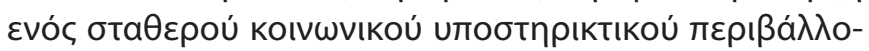
VToc.

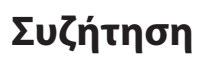

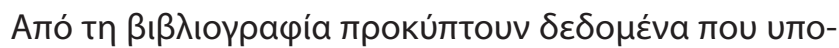

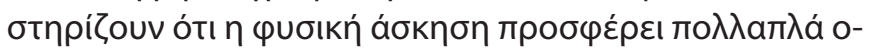

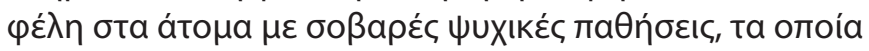
a

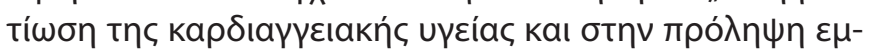

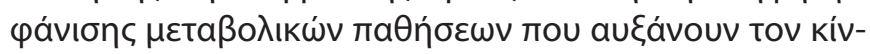

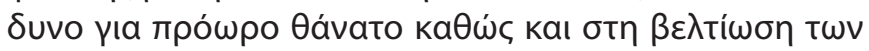

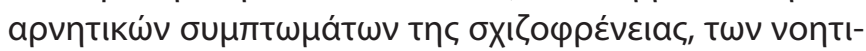

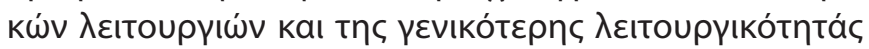

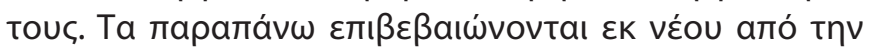

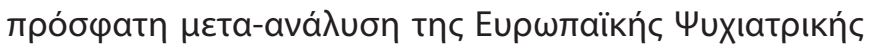

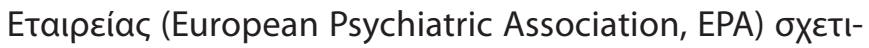

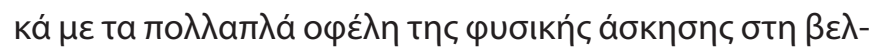

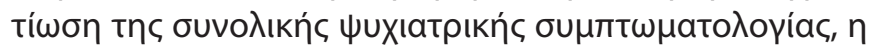

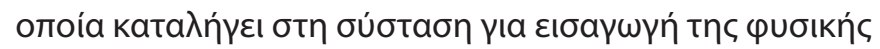

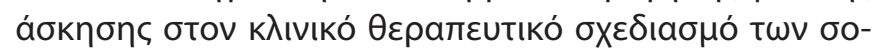

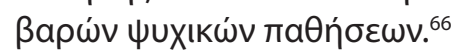

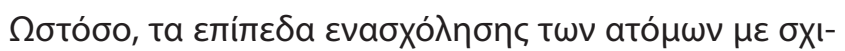

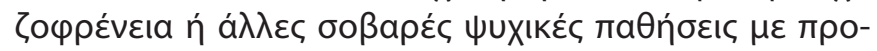

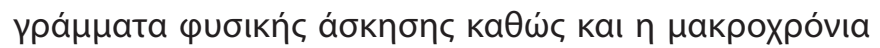

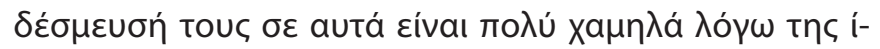

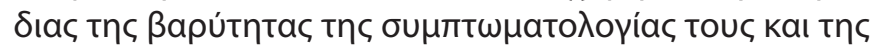

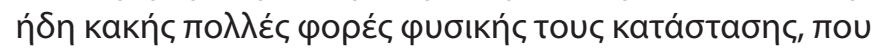

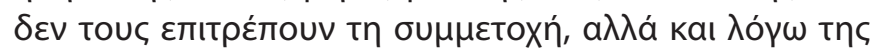

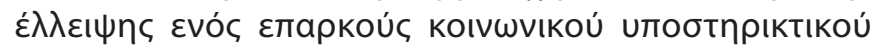

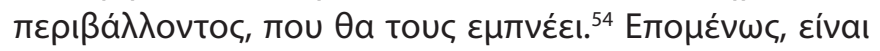

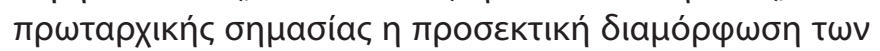

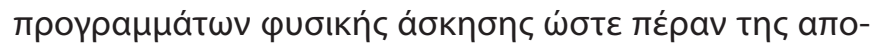

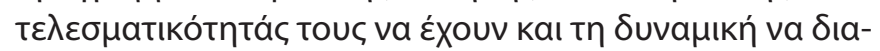

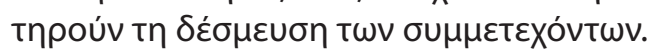

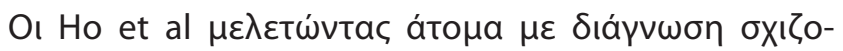

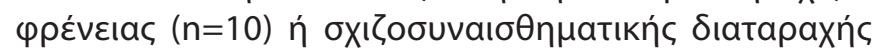

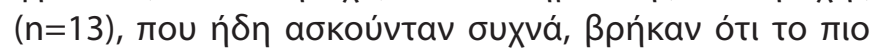

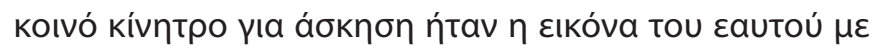

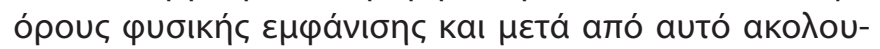

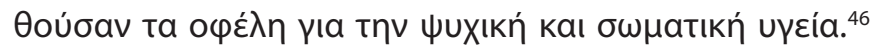

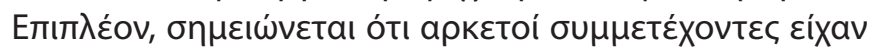

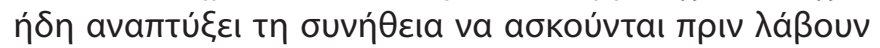

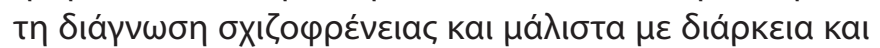

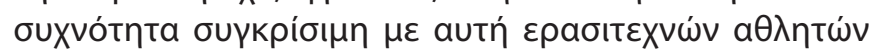

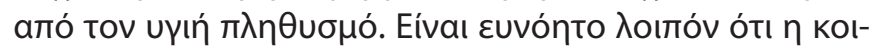

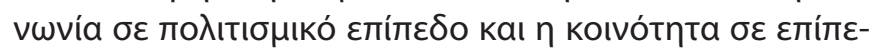

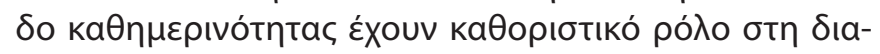

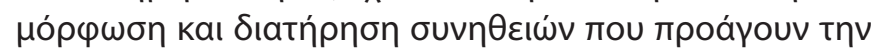

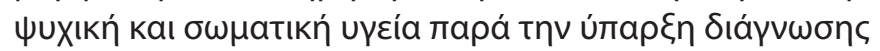

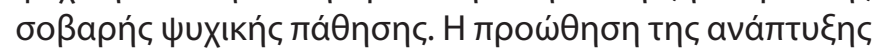

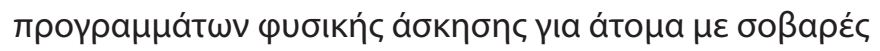

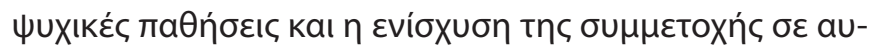

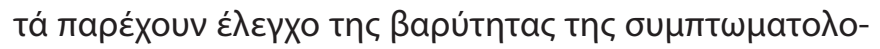

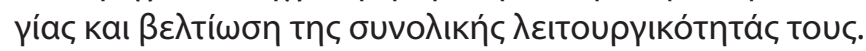

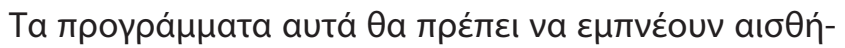

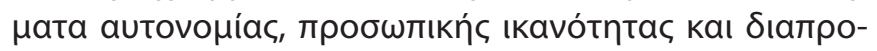

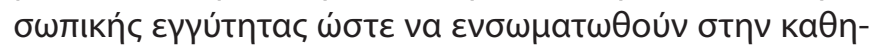

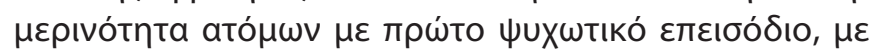

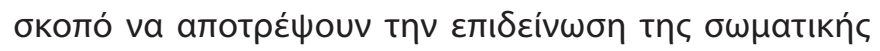

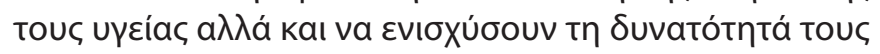

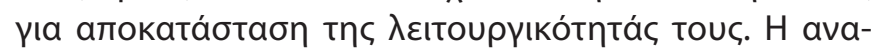

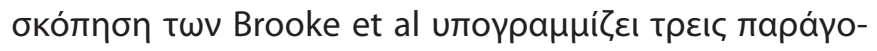

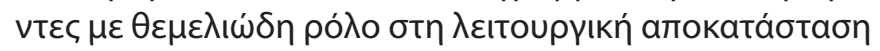

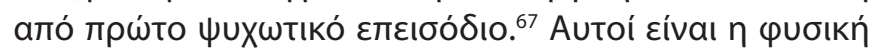

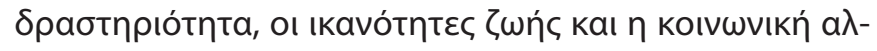

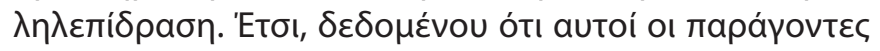

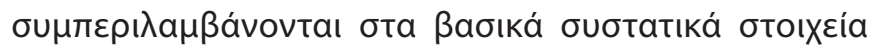

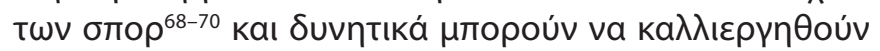




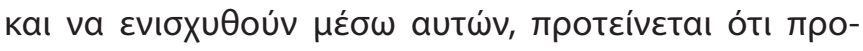

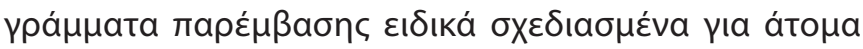

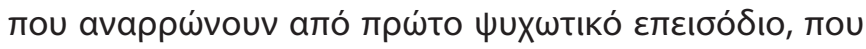

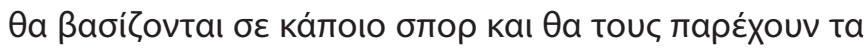

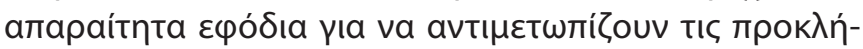

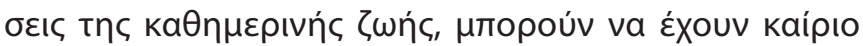

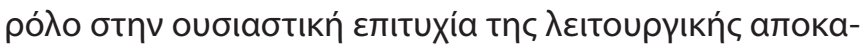

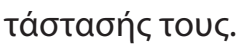

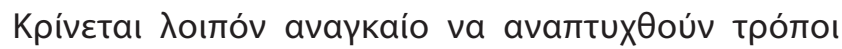

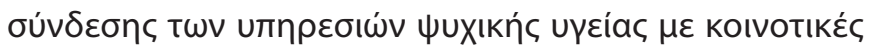

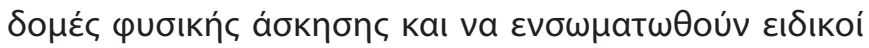

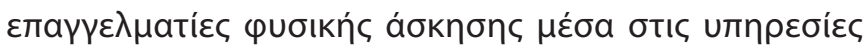

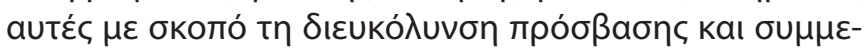

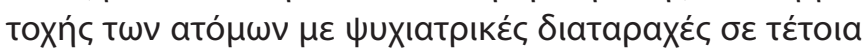

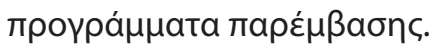

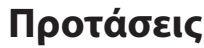

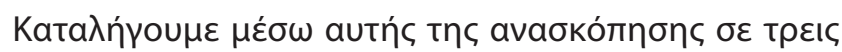

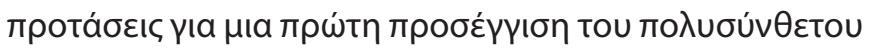

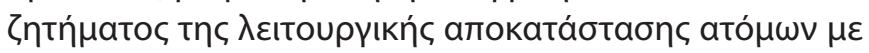

\section{BıBAtoypacpía}

1. Pinckard K, Baskin KK, Stanford KI. Effects of Exercise to Improve Cardiovascular Health. Front Cardiovasc Med 2019, 6:69, doi: 10.3389/ fcvm.2019.00069

2. Dickie K, Micklesfield LK, Chantler S, Lambert EV, Goedecke JH. Cardiorespiratory Fitness and Light-Intensity Physical Activity Are Independently Associated with Reduced Cardiovascular Disease Risk in Urban Black South African Women: A Cross-Sectional Study. Metab Syndr Relat Disord 2016, 14:23-32, doi: 10.1089/met.2015.0064

3. Stewart RA, Benatar J, Maddison R. Living longer by sitting less and moving more. Curr Opin Cardiol 2015, 30:551-557, doi: 10.1097/ HCO.0000000000000207

4. Dickie K, Micklesfield LK, Chantler S, Lambert EV, Goedecke JH. Meeting physical activity guidelines is associated with reduced risk for cardiovascular disease in black South African women; a 5.5-year follow-up study. BMC Public Health 2014, 14:498, doi: 10.1186/14712458-14-498

5. Fiuza-Luces C, Santos-Lozano A, Joyner M, Carrera-Bastos P, Picazo O, Zugaza JL et al. Exercise benefits in cardiovascular disease: beyond attenuation of traditional risk factors. Nat Rev Cardiol 2018, 15:731-743, doi: 10.1038/s41569-018-0065-1

6. Warburton DE, Bredin SS. Reflections on Physical Activity and Health: What Should We Recommend? Can J Cardiol 2016, 32:495-504, doi: 10.1016/j.cjca.2016.01.024

7. Lin X, Zhang X, Guo J, Roberts CK, McKenzie S, Wu WC et al. Effects of Exercise Training on Cardiorespiratory Fitness and Biomarkers of Cardiometabolic Health: A Systematic Review and Meta-Analysis of Randomized Controlled Trials. J Am Heart Assoc 2015, 4. pii: e002014, doi: 10.1161/JAHA.115.002014

8. Khan KM, Thompson AM, Blair SN, Sallis JF, Powell KE, Bull FC. Sport and exercise as contributors to the health of nations. Lancet 2012, 380:59-64, doi: 10.1016/S0140-6736(12)60865-4

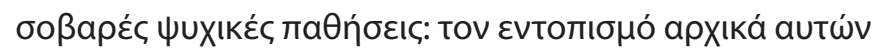

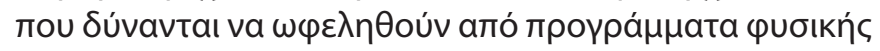

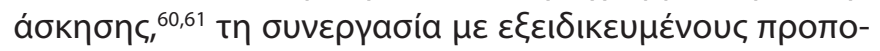

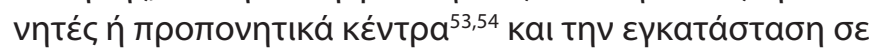

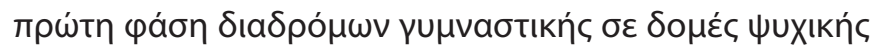

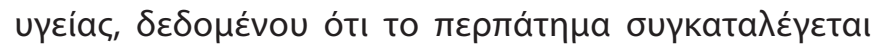

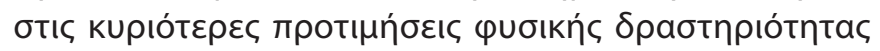

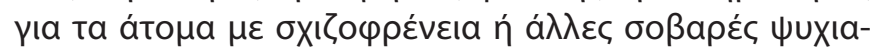

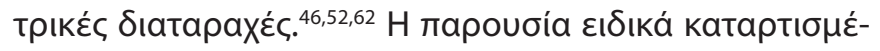

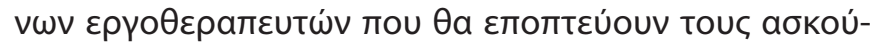

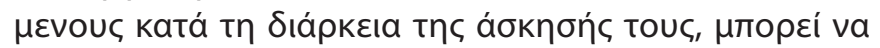

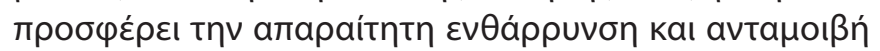

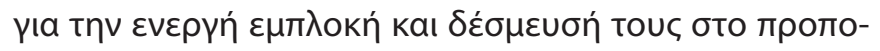

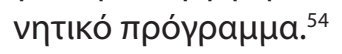

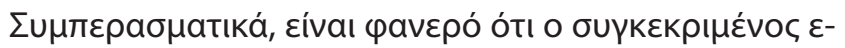

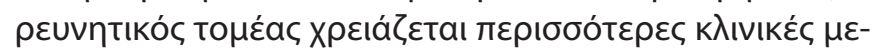

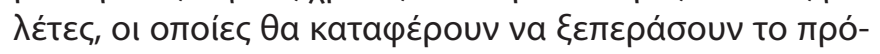

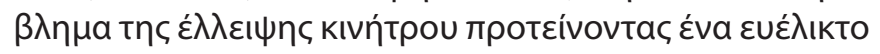

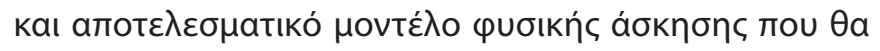

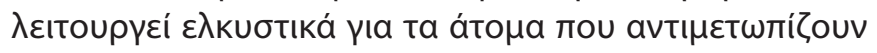

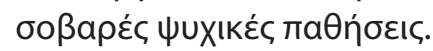

9. Pugh D. Time to encourage patients to take more exercise. Practitioner 2012, 256:25-8,3, PMID: 23252133

10. Sancassiani F, Machado S, Preti A. Physical Activity, Exercise and Sport Programs as Effective Therapeutic Tools in Psychosocial Rehabilitation. Clin Pract Epidemiol Ment Health 2018, 14:6-10, doi: 10.2174/ 1745017901814010006

11. Girdler SJ, Confino JE, Woesner ME. Exercise as a Treatment for Schizophrenia: A Review. Psychopharmacol Bull 2019, 49:56-69, PMID: 30858639

12. Falkai $P$, Malchow $B$, Schmitt A. Aerobic exercise and its effects on cognition in schizophrenia. Curr Opin Psychiatry 2017, 30:171-175, doi: 10.1097/YCO.0000000000000326

13. Nuechterlein KH, Ventura J, McEwen SC, Gretchen-Doorly D, Vinogradov S, Subotnik KL. Enhancing Cognitive Training Through Aerobic Exercise After a First Schizophrenia Episode: Theoretical Conception and Pilot Study. Schizophr Bull 2016, 42(Suppl 1):S44-52, doi: 10.1093/schbul/sbw007

14. Oertel-Knöchel V, Mehler P, Thiel C, Steinbrecher K, Malchow B, Tesky $\checkmark$ et al. Effects of aerobic exercise on cognitive performance and individual psychopathology in depressive and schizophrenia patients. Eur Arch Psychiatry Clin Neurosci 2014, 264:589-604, doi: 10.1007/s00406014-0485-9

15. Matta Mello Portugal E, Cevada T, Sobral Monteiro-Junior R, Teixeira Guimarães T, da Cruz Rubini E, Lattari E et al. Neuroscience of exercise: from neurobiology mechanisms to mental health. Neuropsychobiology 2013, 68:1-14, doi: 10.1159/000350946

16. Pedersen BK, Saltin B. Exercise as medicine - evidence for prescribing exercise as therapy in 26 different chronic diseases. Scand J Med Sci Sports 2015, 25 (Suppl 3):1-72, doi: 10.1111/sms.12581

17. Stanton S, Rosenbaum S, Kalucy M, Reaburn P, Happell B. A call to action: exercise as treatment for patients with mental illness. Aust J Prim Health 2015, 21:120-125, doi: 10.1071/PY14054 
18. Nogueira A, Molinero O, Salguero A, Márquez S. Exercise Addiction in Practitioners of Endurance Sports: A Literature Review. Front Psychol 2018, 9:1484, doi: 10.3389/fpsyg.2018.01484

19. Lichtenstein MB, Hinze CJ, Emborg B, Thomsen F, Hemmingsen SD. Compulsive exercise: links, risks and challenges faced. Psychol Res Behav Manag 2017, 10:85-95, doi: 10.2147/PRBM.S113093

20. Peluso MA, Guerra de Andrade LH. Physical activity and mental health: the association between exercise and mood. Clinics (Sao Paulo) 2005, 60:61-70, doi: 10.1590/s1807-59322005000100012

21. Sommer IE, Kahn RS. The Magic of Movement; the Potential of Exercise to Improve Cognition. Schizophr Bull 2015, 41:776-778, doi: 10.1093/ schbul/sbv031

22. Firth J, Stubbs B, Rosenbaum S, Vancampfort D, Malchow B, Schuch F et al. Aerobic Exercise Improves Cognitive Functioning in People With Schizophrenia: A Systematic Review and Meta-Analysis. Schizophr Bull 2017, 43:546-556, doi: 10.1093/schbul/sbw115

23. Kimhy D, Vakhrusheva J, Bartels MN, Armstrong HF, Ballon JS, Khan S. The Impact of Aerobic Exercise on Brain-Derived Neurotrophic Factor and Neurocognition in Individuals With Schizophrenia: A SingleBlind, Randomized Clinical Trial. Schizophr Bull 2015, 41:859-868, doi: 10.1093/schbul/sbv022

24. Firth J, Stubbs B, Vancampfort D, Schuch F, Lagopoulos J, Rosenbaum $S$ et al. Effect of aerobic exercise on hippocampal volume in humans: A systematic review and meta-analysis. Neuroimage 2018, 166:230238, doi: 10.1016/j.neuroimage.2017.11.007

25. Kandola A, Hendrikse J, Lucassen PJ, Yücel M. Aerobic Exercise as a Tool to Improve Hippocampal Plasticity and Function in Humans: Practical Implications for Mental Health Treatment. Front Hum Neurosci 2016, 10:373, doi: 10.3389/fnhum.2016.00373

26. Pajonk FG, Wobrock T, Gruber O, Scherk H, Berner D, Kaizl I. Hippocampal plasticity in response to exercise in schizophrenia. Arch Gen Psychiatry 2010, 67:133-143, doi: 0.1001/archgenpsychiatry.2009.193

27. Den Ouden L, Kandola A, Suo C, Hendrikse J, Costa RJS, Watt MJ et al. The Influence of Aerobic Exercise on Hippocampal Integrity and Function: Preliminary Findings of a Multi-Modal Imaging Analysis. Brain Plast 2018, 4:211-216, doi: 10.3233/BPL-170053

28. Woodward ML, Gicas KM, Warburton DE, White RF, Rauscher A, Leonova $\mathrm{O}$ et al. Hippocampal volume and vasculature before and after exercise in treatment-resistant schizophrenia. Schizophr Res 2018, 202:158-165, doi: 10.1016/j.schres.2018.06.054

29. Curcic D, Stojmenovic T, Djukic-Dejanovic S, Dikic N, Vesic-Vukasinovic $\mathrm{M}$, Radivojevic $\mathrm{N}$ et al. Positive impact of prescribed physical activity on symptoms of schizophrenia: randomized clinical trial. Psychiatr Danub 2017, 29:459-465, doi: 10.24869/psyd.2017.459

30. Heggelund J, Nilsberg GE, Hoff J, Morken G, Helgerud J. Effects of high aerobic intensity training in patients with schizophrenia: a controlled trial. Nord J Psychiatry 2011, 65:269-275, doi: 10.3109/08039488.2011.560278

31. Wu MH, Lee CP, Hsu SC, Chang CM, Chen CY. Effectiveness of high-intensity interval training on the mental and physical health of people with chronic schizophrenia. Neuropsychiatr Dis Treat 2015, 11:12551263, doi: 10.2147/NDT.S81482

32. Svatkova A, Mandl RC, Scheewe TW, Cahn W, Kahn RS, Hulshoff Pol HE. Physical Exercise Keeps the Brain Connected: Biking Increases White Matter Integrity in Patients With Schizophrenia and Healthy Controls. Schizophr Bull 2015, 41:869-878, doi: 10.1093/schbul/sbv033

33. Schmitt A, Maurus I, Rossner MJ, Röh A, Lembeck M, von Wilmsdorff $M$ et al. Effects of Aerobic Exercise on Metabolic Syndrome, Cardiorespiratory Fitness, and Symptoms in Schizophrenia Include Decreased Mortality. Front Psychiatry 2018, 9:690, doi: 10.3389/fpsyt. 2018.00690
34. Vancampfort D, Rosenbaum S, Schuch F, Ward PB, Richards J, Mugisha $\mathrm{J}$ et al. Cardiorespiratory Fitness in Severe Mental IIIness: A Systematic Review and Meta-analysis. Sports Med 2017, 47:343-352, doi: 10.1007/ s40279-016-0574-1

35. Chalfoun C, Karelis AD, Stip E, Abdel-Baki A. Running for your life: A review of physical activity and cardiovascular disease risk reduction in individuals with schizophrenia. J Sports Sci 2016, 34:1500-1515, doi: 10.1080/02640414.2015.1119875

36. Vakhrusheva J, Marino B, Stroup TS, Kimhy D. Aerobic Exercise in People with Schizophrenia: Neural and Neurocognitive Benefits. Curr Behav Neurosci Rep 2016, 3:165-175, doi: 10.1007/s40473-016-0077-2

37. Dauwan M, Begemann MJ, Heringa SM, Sommer IE. Exercise Improves Clinical Symptoms, Quality of Life, Global Functioning, and Depression in Schizophrenia: A Systematic Review and Meta-analysis. Schizophr Bull 2016, 42:588-599, doi: 10.1093/schbul/sbv164

38. Vancampfort D, Rosenbaum S, Ward PB, Stubbs B. Exercise improves cardiorespiratory fitness in people with schizophrenia: A systematic review and meta-analysis. Schizophr Res 2015, 169:453-457, doi: 10.1016/j.schres.2015.09.029

39. Rosenbaum S, Tiedemann A, Sherrington C, Curtis J, Ward PB. Physical activity interventions for people with mental illness: a systematic review and meta-analysis. J Clin Psychiatry 2014, 75:964-974, doi: 10.4088/JCP.13r08765

40. Firth J, Carney R, Elliott R, French P, Parker S, Mclntyre R et al. Exercise as an intervention for first-episode psychosis: a feasibility study. Early Interv Psychiatry 2018, 12:307-315, doi: 10.1111/eip.12329

41. Spertus J, Horvitz-Lennon M, Abing $H$, Normand SL. Risk of weight gain for specific antipsychotic drugs: a meta-analysis. NPJ Schizophr 2018, 4:12, doi: 10.1038/s41537-018-0053-9

42. Perez-Cruzado D, Cuesta-Vargas A, Vera-Garcia E, Mayoral-Cleries F. Medication and physical activity and physical fitness in severe mental illness. Psychiatry Res 2018, 267:19-24, doi: 10.1016/j.psychres.2018.05.055

43. Wang PW, Lin HC, Su CY, Chen MD, Lin KC, Ko CH et al. Effect of Aerobic Exercise on Improving Symptoms of Individuals With Schizophrenia: A Single Blinded Randomized Control Study. Front Psychiatry 2018, 9:167, doi: 10.3389/fpsyt.2018.00167

44. Khazaeinia T, Ramsey AA, Tam YK. The effects of exercise on the pharmacokinetics of drugs. J Pharm Pharm Sci 2000, 3:292-302, PMID: 11293389

45. McLaughlin M, Jacobs I. Exercise Is Medicine, But Does It Interfere With Medicine? Exerc Sport Sci Rev 2017, 45:127-135, doi: 10.1249/ JES.0000000000000111

46. Ho PA, Dahle DN, Noordsy DL. Why Do People With Schizophrenia Exercise? A Mixed Methods Analysis Among Community Dwelling Regular Exercisers. Front Psychiatry 2018, 9:596, doi: 10.3389/ fpsyt.2018.00596

47. Quirk H, Crank H, Harrop D, Hock E, Copeland R. Understanding the experience of initiating community-based physical activity and social support by people with serious mental illness: a systematic review using a meta-ethnographic approach. Syst $\operatorname{Rev} 2017,6: 214$, doi: 10.1186/s13643-017-0596-2

48. Vancampfort D, Rosenbaum S, Schuch FB, Ward PB, Probst M, Stubbs B. Prevalence and predictors of treatment dropout from physical activity interventions in schizophrenia: a meta-analysis. Gen Hosp Psychiatry 2016, 39:15-23, doi: 10.1016/j.genhosppsych.2015.11.008

49. Farholm A, Sørensen M. Motivation for physical activity and exercise in severe mental illness: A systematic review of cross-sectional studies. Int J Ment Health Nurs 2016, 25:116-126, doi: 10.1111/inm.12217 
50. Vancampfort D, Stubbs B, Venigalla SK, Probst M. Adopting and maintaining physical activity behaviours in people with severe mental illness: The importance of autonomous motivation. Prev Med 2015, 81:216-220, doi: 10.1016/j.ypmed.2015.09.006

51. Bassilios B, Judd F, Pattison P. Why don't people diagnosed with schizophrenia spectrum disorders (SSDs) get enough exercise? Australas Psychiatry 2014, 22:71-77, doi: 10.1177/1039856213510575

52. Ussher M, Stanbury L, Cheeseman V, Faulkner G. Physical activity preferences and perceived barriers to activity among persons with severe mental illness in the United Kingdom. Psychiatr Serv 2007, 58:405-408, doi: 10.1176/ps.2007.58.3.405

53. Firth J, Carney R, French P, Elliott R, Cotter J, Yung AR. Long-term maintenance and effects of exercise in early psychosis. Early Interv Psychiatry 2018, 12:578-585, doi: 10.1111/eip.12365

54. Firth J, Rosenbaum S, Stubbs B, Gorczynski P, Yung AR, Vancampfort D. Motivating factors and barriers towards exercise in severe mental illness: a systematic review and meta-analysis. Psychol Med 2016, 46:2869-2881, doi: 10.1017/S0033291716001732

55. Ryan RM, Deci EL. Self-determination theory and the facilitation of intrinsic motivation, social development, and well-being. Am Psychol 2000, 55:68-78, doi: 10.1037//0003-066x.55.1.68

56. Roman WM, Beebe HL, Burk R. Pilot study provides qualitative evidence for intrinsic motivation in schizophrenia spectrum disorders. OJPsych 2012, 02:321-326, doi: 10.4236/ojpsych.2012.224045

57. Farholm A, Sørensen M, Halvari H. Motivational factors associated with physical activity and quality of life in people with severe mental illness. Scand J Caring Sci 2017, 31:914-921, doi: 10.1111/scs.12413

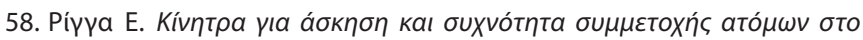

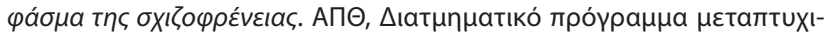

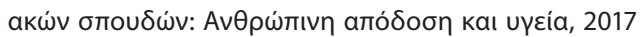

59. Herbsleb M, Schumann A, Malchow B, Puta C, Schulze PC, Gabriel HW et al. Chronotropic incompetence of the heart is associated with exercise intolerance in patients with schizophrenia. Schizophr Res 2018, pii: S0920-9964(18)30094-X, doi: 10.1016/j.schres.2018.02.020

60. Herbsleb M, Keller-Varady K, Wobrock T, Hasan A, Schmitt A, Falkai $P$ et al. The Influence of Continuous Exercising on Chronotropic Incompetence in Multi-Episode Schizophrenia. Front Psychiatry 2019, 10:90, doi: 10.3389/fpsyt.2019.00090
61. Bär KJ. Cardiac Autonomic Dysfunction in Patients with Schizophrenia and Their Healthy Relatives - A Small Review. Front Neurol 2015, 6:139, doi: 10.3389/fneur.2015.00139

62. Subramaniapillai M, Arbour-Nicitopoulos K, Duncan M, McIntyre RS, Mansur RB, Remington $G$ et al. Physical activity preferences of individuals diagnosed with schizophrenia or bipolar disorder. BMC Res Notes 2016, 9:340, doi: 10.1186/s13104-016-2151-y

63. de Vries B, van der Stouwe ECD, Waarheid CO, Poel SHJ, van der Helm EM, Aleman A et al. BEATVIC, a body-oriented resilience therapy using kickboxing exercises for people with a psychotic disorder: a feasibility study. BMC Psychiatry 2018, 18:384, doi: 10.1186/s12888-018-1958-6

64. van der Stouwe EC, de Vries B, Aleman A, Arends J, Waarheid C, Meerdink A et al. BEATVIC, a body-oriented resilience training with elements of kickboxing for individuals with a psychotic disorder: study protocol of a multi-center RCT. BMC Psychiatry 2016, 16:227, doi: 10.1186/s12888-016-0918-2

65. Carless D, Douglas K. The Bristol Active Life Project: Physical Activity and Sport for Mental Health. In: Conrad D, White A (eds) Sports-Based Health Interventions. Springer, New York, 2016

66. Stubbs B, Vancampfort D, Hallgren M, Firth J, Veronese N, Solmi M et al. EPA guidance on physical activity as a treatment for severe mental illness: a meta-review of the evidence and Position Statement from the European Psychiatric Association (EPA), supported by the International Organization of Physical Therapists in Mental Health (IOPTMH). Eur Psychiatry 2018, 54:124-144, doi: 10.1016/j.eurpsy.2018.07.004

67. Brooke LE, Lin A, Ntoumanis N, Gucciardi DF. Is sport an untapped resource for recovery from first episode psychosis? A narrative review and call to action. Early Interv Psychiatry 2019, 13:358-368, doi: 10.1111/ eip. 12720

68. Conrad D, White A. Sports-based health interventions: Case studies from around the world. Springer, New York, 2015, doi: 10.1007/978-1-46145996-5

69. Hermens N, Super S, Verkooijen KT, Koelen MA. A Systematic Review of Life Skill Development Through Sports Programs Serving Socially Vulnerable Youth. Res Q Exerc Sport 2017, 88:408-424, doi: 10.1080/02701367.2017.1355527

70. Soundy A, Freeman P, Stubbs B, Probst M, Roskell C, Vancampfort D. The Psychosocial Consequences of Sports Participation for Individuals with Severe Mental Illness: A Metasynthesis Review. Adv Psychiatry 2015, 1-8, doi: 10.1155/2015/261642 


\title{
Schizophrenia spectrum disorders and physical exercise
}

\author{
Kostas Makris, ${ }^{1}$ Konstantinos Kollias ${ }^{2}$ \\ 'Mobile Mental Health Unit of North-eastern Cyclades, EPAPSY, Patra, \\ ${ }^{2}$ National and Kapodestrian University of Athens, Medical School, First Department of Psychiatry, Eginition Hospital, Athens, Greece
}

ARTICLE HISTORY: Received 5 November 2019 / Revised 20 February 2020 / Published Online 28 May 2021

\begin{abstract}
Recent research data points to the beneficial role of physical exercise in mental health. Long-term physical activity may promote neurogenesis and angiogenesis in people with mental illnesses improving their cognitive functions and overall functionality. In many cases, it is strongly recommended to include physical exercise in the therapeutic planning for people with mental illnesses, including schizophrenia. The purpose of this review is to study current research data on the effect of physical exercise on the symptomatology of the first-episode psychosis and schizophrenia as well as to search for any physical exercise programs suitable for the mentally ill. International literature as well as Greek online publications were searched. The search language was English and the search terms were: "schizophrenia", "first episode psychosis", "physical exercise". A study of the literature suggests that physical exercise offers multiple benefits to people with severe mental illness, such as weight control, improved cardiovascular health, preventing metabolic disorders - with the positive implications of the above in relation to receiving antipsychotic treatment- and improving the negative symptoms of schizophrenia, cognitive functioning and global functioning by activating the neuroplasticity of the brain. However, starting and engaging in physical activity programs faces many obstacles for the population under study. It appears that when the physical activity programs offered give participants both pleasure and satisfaction, promote personal choice of physical activity and the opportunity for group interaction during the process, then the likelihood of commitment to them is maximized. The crucial importance of a stable social-supportive environment for the success of intervention programs is mentioned. In addition, there is a need to develop ways of linking mental health services with community physical training centres and incorporating specialized professional coaches into these services. Moreover, this area of research needs further clinical studies to develop more effective training programs that overcome the lack of motivation experienced by people with severe mental illness. Installing treadmills in mental health centres, establishing partnerships with specialized coaches or community training centres and identifying individuals who may benefit from physical exercise programs are some of the first steps addressing the matter of functional recovery from first-episode psychosis and schizophrenia.
\end{abstract}

KEYWORDS: Schizophrenia, first episode psychosis, physical exercise. 